\title{
The successful treatment of vocal cord dysfunction with low-dose amitriptyline - including literature review
}

\author{
VA Varney' \\ H Parnell' \\ J Evans' \\ NT Cooke' \\ J Lloyd ${ }^{2}$ \\ J Bolton ${ }^{3}$ \\ 'Department of Respiratory Medicine, \\ ${ }^{2}$ Department of Speech and Language \\ Therapy, ${ }^{3}$ Department of Liaison \\ Psychiatry, St Helier Hospital, \\ Carshalton, Surrey, UK
}

Correspondence: VA Varney Department of Respiratory Medicine, St Helier Hospital,Wrythe Lane, Carshalton, Surrey SM5 IAA, UK Email veronica.varney@esth.nhs.uk
This article was published in the following Dove Press journal:

Journal of Asthma and Allergy

18 November 2009

Number of times this article has been viewed

\begin{abstract}
Vocal cord dysfunction is an asthma mimic. Diagnosis of this condition requires a high index of suspicion if unnecessary treatments are to be avoided. We describe the findings from our case series of 62 patients (age range 18 to 90 years) in whom the diagnosis was confirmed. Our findings show low-dose amitriptyline to be very effective in $90 \%$ of cases, with rapid benefit for those patients whose symptoms had been present for less than 12 months. This treatment, in conjunction with psycho-therapeutic and behavioral therapies may reduce unnecessary hospital admissions. Future studies may show whether this treatment regimen may reduce demands on the speech and language therapists.
\end{abstract}

Keywords: vocal cord dysfunction, asthma, amitriptyline, wheeze, anxiety

\section{Introduction}

Vocal cord dysfunction (VCD) is an asthma mimic, which may present in association with asthma or be misdiagnosed as asthma. ${ }^{1,2}$ The literature reports an average delay of 4 years to the correct diagnosis in such cases. ${ }^{3}$ There is a female predominance, ${ }^{4}$ with quoted ratios of 40 female: 1 male. ${ }^{5} 81 \%$ of patients are Caucasian with a common age range of 14 to 60 years.

Diagnosis requires a high index of suspicion and the following factors may hold clues to determining a correct diagnosis: $:^{1,3,6}$

a) Suspect if asthma suddenly deteriorates or becomes unresponsive to previous treatments.

b) Attacks of wheezing worse by day, which may be surprisingly short lived. ${ }^{5}$

c) Steroid "pseudo-resistance" is demonstrated; where patients previously responsive to steroids appear to no longer respond.

d) Attacks that are provoked by exercise. ${ }^{5}$

e) Pungent smells, hyperventilation, stress or cough may be precipitants of an attack of wheeze. ${ }^{7}$

The literature recognizes 3 patterns of VCD

1. Exercise-induced VCD where symptoms are limited to exercise.

2. Spontaneous VCD where there is a sudden onset of symptoms without exercise.

3. Persistent VCD where adduction of the vocal cords in both inspiration and expiration is observed. This is usually associated with an emergency admission.

Observation or history taking may reveal an attack of sudden onset occurring spontaneously or with exercise and of a brief duration. There may be tightness in the throat, with a flexed neck during attacks. A change in voice quality, due to misuse of the vocal 
cords may be present. Attempts to measure lung function may show variable results, depending on the degree of vocal cord adduction during each measurement. ${ }^{8}$ If the vocal cord adduction is severe an intense audible stridor, which is predominantly inspiratory can be heard. This would suggest that persistent cord adduction is occurring. Asymptomatic periods may occur. Chest tightness and breathlessness on exertion, with a cough would suggest exercise-induced VCD. This often occurs at a set distance. These distances can be very short, sometimes $<10 \mathrm{~m}$. There may be a feeling of trapped mucus around the vocal cord area, resulting from the interruption of the normal mucociliary clearance due to cord adduction.

In normal movement of the vocal cords, there is inspiratory abduction (opening) followed by a brief expiratory phase adduction (closing) of $2 \mathrm{~mm}$ from the fully abducted position to generate physiological autoPEEP. ${ }^{6,9}$ This is the positive end expiratory pressure generated in part by the vocal cords to prevent early airway closure. The cords then open again for inspiration. Voluntary cord adduction in a sustained manner during inspiration is impossible in the healthy normal subject. ${ }^{6,7}$ Abnormal inspiratory vocal cord adduction may have pathological (cerebral) causes such as Arnold-Chiari malformation and cerebral aqueduct stenosis or even drug induced brain stem dysfunction. This has also been noted in motor neurone disease, myotonic disorders, Parkinson's disease and laryngeal breathing dystonias. ${ }^{7}$

In non-organic VCD, inspiratory adduction of the anterior two-thirds of the cords can produce a diamond-shaped opening in the posterior segment. ${ }^{9}$ This can generate the pitch of wheeze similar to asthma, but the wheeze is loudest over the larynx and is usually audible. ${ }^{8}$ The chest $\mathrm{x}$-ray is often normal, but some patients with persistent adduction can induce acute hyperinflation and use accessory muscles, if adduction is severe. ${ }^{1}$ Presentation to intensive treatment unit in status asthmaticus, even with a silent chest, has been described. Intubation often finds normal airway pressures and therefore extubation usually follows within a short time. In the acute setting of possible status asthmaticus, it is difficult to take a different course of action. Once recognized, it is appropriate for these patients to be treated with heliox (a mixture of oxygen 24\% and helium) and light anxiolytics. ${ }^{1,7}$

The results of measured lung function are variable, ${ }^{3}$ with obstructive, restrictive or normal values appearing, depending upon the activity of the VCD. Arterial blood gases are usually normal, unless persistent adduction of the vocal cords occurs in which a silent chest may be produced. ${ }^{3,8}$
Failure to recognize the condition of VCD, leads to the prescription of unnecessary medication with associated adverse effects. ${ }^{1,2}$ Repeat admissions for what is a benign condition is undesirable and reinforces the undiagnosed problem. ${ }^{10}$

Physicians managing patients with VCD recognize the importance of psychological stresses on clinical symptoms. ${ }^{3,11}$ VCD is associated with a high rate of co-morbid psychiatric disorders. One survey found that $73 \%$ of 95 patients with VCD had a psychiatric diagnosis. ${ }^{8,12,14}$ It has also been suggested that patients with this condition have an increased rate of childhood trauma or abuse. , $^{1,4}$

Treatment to relieve symptoms is recommended before attempting to deal with the psychological stress. ${ }^{89}$ Without this symptom relief, addressing psychological conditions can aggravate vocal cord dysfunction. Treatments to date include: ${ }^{4}$ "panting" and other behavioral techniques to encourage abduction of the vocal cords, ${ }^{2,9}$ with such patient education performed by a speech and language therapist. ${ }^{7}$ Tranquillizers are also recommended. ${ }^{3}$ The literature describes variable resistance breathing systems which consists of facemasks with one-way valves to induce inspiratory drag. ${ }^{13}$ This reduces inspiratory stridor and gives psychological relief, which may reduce reinforcement of the attack and offer a solution. Historical treatments included tracheostomy. ${ }^{6}$ Botulinum injections have been proposed as a treatment, but no randomized controlled studies have yet been published. ${ }^{5,7}$

Examination of vocal cords in the absence of symptoms may show normal movements. However, in persistent VCD the vocal cord adduction may be seen at any time. ${ }^{7}$ Breathing is often reported by relatives to improve during sleep and to deteriorate when mental stress occurs. The condition itself, if unrecognized, leads to increased anxiety and a consequent increase in the VCD severity. Some patients may show more than one of the 3 patterns of VCD described. Without a high index of suspicion, the condition may be overlooked in asthmatics. ${ }^{4}$ A routine auscultation over the vocal cord area is a useful screen during acute admissions and outpatient reviews. It is especially indicated if asthma becomes worse or appears steroid unresponsive. ${ }^{10}$ Even $\mathrm{B}_{2}$ agonists can aggravate symptoms in individuals with VCD, a feature not noted frequently in true asthma attacks. During vocal cord inspection, if adduction of the cords is visible only in expiration, this must be interpreted with caution, as it is likely to be a physiological and adaptive mechanisms and not VCD. ${ }^{7}$ The paradoxical movements must be those of inspiratory adduction. ${ }^{6}$ If the flow volume loops are measured during an attack abnormalities will 
be demonstrated. ${ }^{2}$ Twenty-five percent of subjects may show variable but persistently abnormal loops. In these patients, extra thoracic obstruction must be excluded. Fifty percent of subjects can have VCD by exercise. ${ }^{5,7}$

It is important for the respiratory technician to print all flow-volume loops, as there is a risk that any loops of an unusual appearance, may be disregarded as an error and not be recognized as related to VCD. Flutter of the inspiratory loop and brief changes in the expiratory loop causing flattening (top hat effect) are described. ${ }^{1}$ From the flow volume loop, visible flattening of the inspiratory portion is seen and indicates upper airway obstruction, which could include other causes such as subglottic stenosis and vocal cord dysfunction. This makes inspection of the cords essential. From the flow-volume loop, if the maximum mid expiratory flow is divided by the maximum mid inspiratory flow and has a value of $>1.5$ this suggests vocal cord dysfunction. ${ }^{2}$

\section{Method}

\section{The patients}

The study describes 62 consecutive patients. Age range 18 to 90 years ( 17 male/45 female) in whom VCD was suspected and confirmed. In $40 \%$, previous stable asthma had been present but a change in clinical circumstances had occurred. This had taken the form of treatment resistance, or hospital admission for an asthma attack. Others were referred to outpatients with a new suspected asthma, which appeared unresponsive to their GP's treatment.

\section{History and physical findings}

A history of asthma-like symptoms was sought and past asthma patterns established with spirometry and peak flows recorded. Skin tests for atopy were performed if atopic symptoms were present. Specific enquiry was made into possible symptoms of VCD. These included frequent attacks of audible wheezy noises (or stridor), occurring during exercise or associated with stress, or brief daytime attacks with or without recognized triggers. Voice changes, throat tightness and a feeling of mucus adhering around the vocal cord area were enquired about. The limitation of exercise and the walking distance before symptoms occurred were established. A walking test was performed, so that observation and auscultation of the chest and vocal cord area could be performed.

\section{Anxiety and assessment}

Although a formal assessment of mood was not undertaken, recent life events were discussed, including enquires about any work stress or trauma that may have preceded the symptoms. Significant insomnia was found in $95 \%$ of confirmed cases.

\section{Fiber-optic examination of the cords}

This was undertaken for each case, with attempts to establish likely triggers before the examination. This would allow examination of the cords, followed by "a stress" to induce VCD. In a large number of cases the "stress" was through exercise, especially climbing stairs. In others cases, pungent smells, including perfume, brought by the patients to the fiberoptic test were used as triggers. Many were induced by the lignocaine preparation for the fiber-optic examination and the anxiety associated with the investigation. Careful inspection of the cords for vocal cord disease, especially tumors and paralysis was as important as assessing movement. Two patients with vocal cord cancers were identified, and excluded from this study however this emphasized the importance of inspecting all cords. The cord movement was inspected during the induced wheeze/stridor when the patient was asked to take deep breaths in and out despite their condition. The inspiratory and expiratory phase of respiration was determined by a hand placed on the sternum, so that simultaneous cord inspection and the phase of respiration were accurately assessed. Adduction during the inspiratory phase was confirmation of vocal cord dysfunction. Acid reflux around the epiglottis and vocal cord area was also noted and treated if present.

\section{Methacholine test}

Patients with a significant history of asthma and vocal cord symptoms had a methacholine test to assess bronchial hyperreactivity, if it was doubtful that their inhaled therapy was optimal.

\section{Amitriptyline treatment}

Tricyclic antidepressants have no previously described benefit in VCD. However, the authors had noted that a low dose of amitriptline for night sedation, often led to a rapid improvement in the symptoms of VCD. The starting doses were low, $10 \mathrm{mg} 2$ to 3 hours before bed, which were increased by $10 \mathrm{mg}$ each week until adequate sleep was induced. Many of the patients in this series were already on long term selective serotonin reuptake inhibitors (SSRIs). If this was the case, the SSRI was maintained and amitriptyline was added to it. Patients with protracted symptoms with treatment resistance and anxiety affecting their quality of life were referred to liaison psychiatry. During the course of our investigations, if the patients were judged to have previously 
undiagnosed anxiety related or depressive illness, they were referred to the local liaison psychiatry service for further management.

\section{Referral to speech therapy}

There is a high prevalence of abnormal voice quality in vocal cord dysfunction ${ }^{15}$ and behavioral treatment offered by voice specialist speech and language therapists (SLT, speech pathologists) is an encouraging option. ${ }^{16}$ Significant subjective and objective improvements in symptoms have been recorded in studies of the characteristics of laryngeal respiratory disorders and their treatment, via a combination of medical management and respiratory retraining. ${ }^{17,18}$

In the study, 43 patients were referred to the SLT. Those who had not previously had their vocal cords examined were seen for nasoendoscopy with the ear-nose-throat (ENT) surgeon in the joint ENT/SLT clinic for further assessment of laryngeal structure and function.

Therapeutic approaches were predicated on explanatory discussion of laryngeal mechanisms and vocal cord function in normal respiration and phonation. The causes and effects of abnormal/paradoxical vocal cord movement were discussed in relation to patients' presenting symptoms. Possible chemical triggers and psychosocial aspects were also discussed. Patients benefited from reassurance that VCD is caused by central chemo-reflex changes, which can be reversible via behavioral techniques.

Specific therapeutic techniques aimed to encourage vocal cord abduction and to divert attention away from the larynx included:

- Sip and swallow routines.

- Pursed lip breathing.

- Blowing against finger held to the lips.

- Nasal only breathing.

- Abdominal breathing.

The success of treatment depended heavily upon patients' response to the opportunity to discuss and understand their symptoms and to bring them under voluntary control.

Patients who did not benefit from voice therapy were those who, at the time of treatment, were unable to meet the challenge of changing maladaptive respiratory behaviors.

\section{Results}

Table 1 shows the patients' demographics. All patients had VCD confirmed at fiber-optic examination. Two patients had vocal cord tumors and are excluded from further analysis.

In the case series, previous asthma was higher in women (64\%) than men (31\%). All patients described significant
Table I Patient demographics: the case series and the percentage with prior asthma, insomnia, and anxiety at diagnosis

\begin{tabular}{lll}
\hline & Male & Female \\
\hline Number of subjects & 17 & 45 \\
Average age (range) & $54(19-80)$ & $48(8-90)$ \\
Previous asthma & $31 \%$ & $64 \%$ \\
$\%$ with insomnia & $95 \%$ & $91 \%$ \\
$\%$ aware of anxiety & $31 \%$ & $72 \%$ \\
$\%$ with traumatic life event/work stress & $41 \%$ & $32 \%$ \\
$\%$ on antidepressant treatment & $12 \%$ & $65 \%$ \\
at presentation & & \\
$\%$ with spontaneous and persistent & $7 \%$ & $16 \%$ \\
VCD symptoms & & \\
\hline
\end{tabular}

Abbreviation: VCD, vocal cord dysfunction.

insomnia and awareness of anxiety symptoms (including panic) which was twice as common in women (72\%) than men (31\%). Life events and work stress affected up to $40 \%$ of the series with $65 \%$ of women already prescribed an antidepressant.

Most patients' symptoms were intermittent or exerciseinduced but $16 \%$ were more persistent and appeared a feature in older women with chronic asthma and anxiety. Amitriptyline had beneficial effects on insomnia and improved reported anxiety particularly in men.

The response to amitriptyline and the mean dose required is shown in Table 2.

There was an identical approach to treatment in all patients. This involved initially starting a $10 \mathrm{mg}$ dose of amitriptyline 2 hours before bed for the first week. Patients were instructed to increase the dose by $10 \mathrm{mg}$ each week (maximum dose $75 \mathrm{mg}$ ) until sleep was restored. Once sleep improved, VCD appeared to show rapid improvement in

Table 2 The response to amitriptyline: responses and doses of amitriptyline required and its benefit, treatment failures and sideeffects

\begin{tabular}{lll}
\hline & Male & Female \\
\hline $\begin{array}{l}\text { Number of subjects } \\
\text { \% responding to amitriptyline }\end{array}$ & 17 & 45 \\
with cessation of VCD & $94 \%$ & $82 \%$ \\
$\begin{array}{l}\text { Mean dose required (range) } \\
\% \text { with improved insomnia }\end{array}$ & $20 \mathrm{mg}(10-40)$ & $20 \mathrm{mg}(10-75)$ \\
$\%$ with improved anxiety & $100 \%$ & $100 \%$ \\
$\begin{array}{l}\text { Treatment failures where VCD } \\
\text { did not cease }\end{array}$ & $90 \%$ & $96 \%$ \\
Intolerance due to side effects & $\mathrm{Nil}$ & $18 \%$ \\
\hline
\end{tabular}

Abbreviation: VCD, vocal cord dysfunction. 
most patients. We learnt that inspection of the cords was necessary before treatment, as many patients' symptoms had resolved from the start of treatment and before the fiber-optic examination just 5 to 7 days later.

The response to amitriptyline was higher in men (94\%) than women $(82 \%)$, but insomnia improved in all patients. Anxiety also improved in $>90 \%$ of patients. Eight patients who had long-standing persistent VCD, which had not been recognized early, were difficult clinical management problems. While they did show some improvement with amitriptyline, the VCD did not cease even though its severity did diminish. In these patients help with liaison psychiatry was necessary.

The response to amitriptyline was sufficient on a mean dose of $20 \mathrm{mg}$, which is low enough to not have significant side-effects or interaction with other ongoing antidepressant therapy. Some patients did require a full dose of $75 \mathrm{mg}$ for benefit, but this was the minority. We found that many patients once improved, were able to reduce the dose of amitriptyline within a few weeks to $10 \mathrm{mg}$ without relapse, and continued this dose for the minimum 6-month period suggested by us. Re-enforcing the nature of the condition and demonstrating the paradoxical movement of the cords at fiber-optic examination did prove helpful in allowing the patient to take charge of the condition with advice from the SLT.

\section{Adverse effects of amitriptyline and duration of treatment}

The initial dose was low (10 mg), in order to avoid over sedation and a dry mouth. The treatment period was 3 to 6 months to establish new vocal cord patterns and break old habits. The mean dose required was $20 \mathrm{mg}$ at bedtime, but the range of doses used is shown in Table 2 .

\section{Discussion}

This case series demonstrated a reproducible benefit of low-dose amitriptyline in patients with VCD. The mean dose required was $20 \mathrm{mg}$ taken 2 to 3 hours before bed. In the majority of patients this resulted in symptom resolution within 1 week. At outpatient review at 4 weeks there was resolution of the problem in most cases with reduced anxiety and greatly improved length and quality of sleep. The high percentage of patients already taking SSRIs indicated that the presence of emotional problems had been recognized, but these drugs did not appear to influence VCD. The addition of low-dose amitriptyline, without withdrawal of the SSRI, did not lead to therapeutic problems. It became clear from the series that resolution was quickest in those with a shorter duration of VCD (less than 3 months), where symptoms could abate in 1 to 7 days regardless of prior asthma. This emphasizes the importance issue of inspection of the cords before commencing treatment and early recognition to confirm the diagnosis before starting treatment. In patients with the condition for 3 to 12 months prior to diagnosis, resolution was likely by 4 weeks. There were few treatment failures, but when they did occur the patients were generally female and older ( $>60$ years) with co-morbid asthma and psychological problems. Spontaneous attacks and exerciseinduced vocal cord symptoms appeared to resolve well with a longer duration of amitriptyline

Insomnia, which was significant, was present in over $90 \%$ of all cases of proven VCD and appeared to be associated with the condition. Questioning patients can frequently define an initiating stress in their life, particularly in those without prior difficult asthma.

Referral to speech and language therapy is extremely helpful, but resources are limited and regional waiting times vary. Therefore the treatment with amitriptyline could serve to indicate more precisely those patients who would benefit more from such therapy. This series demonstrated that the age range of patients is greater than that published in the literature, with cases occurring into the 90th year often following traumatic events.

Our findings suggest that low-dose amitriptyline (10 to $20 \mathrm{mg}$ ) could be a cost effective treatment in patients with VCD. Initial treatment, even in mild cases needs to be for 3 to 6 months. This should re-establish a healthy vocal cord pattern. In other cases of more persistent VCD, indefinite prophylactic treatment may be warranted with the aim of improving quality of life and avoiding unnecessary hospital admissions and treatments.

This case series is the first to identify the use of amitriptyline in VCD, but clearly this is not a randomized controlled trial and did not formally measure psychiatric morbidities in each patient. The severity of asthma was assessed, but not by a standardized protocol.

Although the study indicated that low-dose amitriptyline is associated with an improvement in the symptoms of VCD, the mechanism of action is unknown. Amitriptyline was prescribed in doses that are generally sub therapeutic for anxiety and depressive disorders, yet sleep benefit with a secondary effect on the cords was observed. It may act directly on the nervous control of the cords. Low-dose amitriptyline is commonly used by the rheumatologists to treat neck spasms and headaches. The drug is known 
to have several sites of action in the nervous system, one of which may include muscle relaxation. VCD has been described in 2 case reports after prolonged stimulation of the vagal nerve by implanted devices. This suggested that the vagus may have a role in the condition. Clinical benefit has been described in 6 patients with exercise-induced VCD who inhaled the anticholinergic drug ipratropium prior to exercise. ${ }^{19,20}$ It is known that amitriptyline has anticholinergic affects also.

The findings would support the need for a randomized controlled trial to fully assess an active and control arm. This could include stratification of the VCD to intermittent, frequent and severe. It could also assess fully, anxiety levels, asthma status, life events and outcomes in the active and control groups.

\section{Disclosures}

The authors report no conflict of interest in the submitted manuscript.

\section{References}

1. Murray DM, Lawler PG. All that wheezes is not asthma. Anaesthesia. 1998;53:1002-1011.

2. Borer H, Hänni P, Schoenenberger RA. Vocal cord dysfunction: an important differential diagnosis of brittle asthma. Respiration. 2001;68:318.

3. Wolfe JM, Meth BM. Vocal cord dysfunction mimiking a severe asthma attack. J Emerg Med. 1999;17:39-41.

4. Tan K L, Eng P, Ong UYY. Vocal cord dysfunction: two case reports. Ann Acad Med Singapore. 1997;26:494-496.

5. Levin M. Vocal cord dysfunction complicating asthma. Curr Allergy Clin Immunol. 2003;16:12-14.
6. Bahrainwala H, Simon MR, Harrison DD, Toder D, Secord EA. A typical expiratory flow volume curve in an asthmatic patient with vocal cord dysfunction. Ann Allergy Asthma Immunol. 2001;86: 439-443.

7. Bahrainwala AH, Simon MR. Wheezing and vocal cord dysfunction mimiking asthma. Curr Opin Pulm Med. 2001;7:8-13.

8. McQuaid EL, Spieth LE, Spirito A. Vocal-cord dysfunction presenting as asthma. J Pediatr Psychol. 1997;22:739-748.

9. Place R, Morrison A, Arce EM. Vocal cord dysfunction. J Adolesc Health. 2000;27:125-129.

10. Roberts NJ, Robinson DS, Partridge MR. How is difficult asthma managed? Eur Respir J. 2006;28:968-973.

11. Gavin LA, Wamboldt M, Brugman S, Roesler TA, Wamboldt F. Psychological and family characteristics of adolescents with vocal cord dysfunction. J Asthma. 1998;35:409-417.

12. Newman KB, Mason VG, Schmaling KB. Clinical features of vocal cord dysfunction. Am J Res Crit Care Med. 1995;152:1382-1386.

13. Archer GI, Hoyle JL, McClusky A, Macdonald J. Inspiratory vocal cord dysfunction and new approach in treatment. Eur Respir J. 2000; 15:617-618.

14. Freedman MR, Rosenberg SJ, Schmaling KB. Childhood sexual abuse in patients vocal cord dysfunction. J Nerv Ment Dis. 1991;179: 295-298.

15. Vertigan AE, Theodoros DG, Winkworth AL. Perceptual voice characteristics in chronic cough and paradoxical vocal fold movement. Folia phoniatrica et logopaedica. 2007;59:256-267.

16. Vertigan AE, Theodoros DG, Gibson PG, Winkworth AL. The relationship between chronic cough and paradoxical vocal cord movement: A review of the literature. $J$ Voice. 2006;20:466-480.

17. Murray T, Tabaee A, Owezarzak V, Aviv JE. Respiratory retraining therapy and management of laryngopharangeal reflux in the treatment of patients with chronic cough and paradoxical vocal fold movement disorder. Ann Otol Rhinol Laryngol. 2006;115:754-758.

18. Vertigan AE, Theodoros DG, Gibson PG, Winkworth AL. Voice and upper airway symptoms in people with chronic cough and paradoxical vocal fold movement. $J$ Voice. 2007;21:361-383.

19. Devang R,Weinberger M. Longterm outcome of vocal cord dysfunction. Ann Allergy Asthma Immunol. 2006;96:794-799.

20. Weinberger M, Abu-Hasan M. Pseudo-asthma: when cough, wheezing and dyspnoea are not asthma. Pediatrics. 2007;120:855-864.

\section{Publish your work in this journal}

The Journal of Asthma and Allergy is an international, peer-reviewed open-access journal publishing original research, reports, editorials and commentaries on the following topics: Asthma; Pulmonary physiology; Asthma related clinical health; Clinical immunology and the immunological basis of disease; Pharmacological interventions and

\section{Dovepress}

new therapies. Issues of patient safety and quality of care will also be considered. The manuscript management system is completely online and includes a very quick and fair peer-review system, which is all easy to use. Visit http://www.dovepress.com/testimonials.php to read real quotes from published authors. 